\title{
Severe nausea and vomiting in pregnancy: psychiatric and cognitive problems and brain structure in children
}

Hui Wang ${ }^{1 \dagger}$, Edmund T. Rolls $s^{2,4,5 \dagger}$, Xiujuan Du', Jingnan Dư ${ }^{2}$, Dexin Yang ${ }^{2}$, Jiong Li ${ }^{1,6}$, Fei $\mathrm{Li}^{i^{*}}$, Wei Cheng ${ }^{2,3^{*}}$ and Jianfeng Feng $2,3,4$

\begin{abstract}
Background: Two studies have suggested that severe prolonged nausea and vomiting during pregnancy is associated with emotional and behavioral problems in offspring, with smaller sample size and short-term follow-up. Moreover, little information is available on the role of the brain structure in the associations.

Methods: In a US-based cohort, the association was investigated between severe prolonged nausea and vomiting in pregnancy (extending after the second trimester and termed SNVP), psychiatric and cognitive problems, and brain morphology, from the Adolescent Brain Cognitive Development (ABCD) study, from 10,710 children aged 9-11 years. We validated the emotional including psychiatric findings using the Danish National Cohort Study with 2,092,897 participants.

Results: SNVP was significantly associated with emotional and psychiatric problems ( $t=8.89$, Cohen's $d=0.172, p=$ $\left.6.9 \times 10^{-19}\right)$ and reduced global cognitive performance $\left(t=-4.34, d=-0.085, p=1.4 \times 10^{-5}\right)$ in children. SNVP was associated with low cortical area and volume, especially in the cingulate cortex, precuneus, and superior medial prefrontal cortex. These lower cortical areas and volumes significantly mediated the relation between SNVP and the psychiatric and cognitive problems in children. In the Danish National Cohort, severe nausea and vomiting in pregnancy were significantly associated with increased risks of behavioral and emotional disorders in children (hazard ratio, 1.24; 95\% confidence interval, 1.16-1.33).

Conclusions: SNVP is strongly associated with psychiatric and cognitive problems in children, with mediation by brain structure. These associations highlight the clinical importance and potential benefits of the treatment of SNVP, which could reduce the risk of psychiatric disorder in the next generation.
\end{abstract}

Keywords: Nausea and vomiting, Cognitive performance, Psychiatric problems, Cortical structure, Cingulate cortex, Precuneus, Superior medial prefrontal cortex

\footnotetext{
* Correspondence: feili@shsmu.edu.cn; wcheng@fudan.edu.cn

${ }^{+}$Hui Wang and Edmund T. Rolls contributed equally to the study and considered are the co-first authors.

'Department of Developmental and Behavioral Pediatric \& Child Primary Care/MOE-Shanghai Key Laboratory of Children's Environmental Health, Xin Hua Hospital Affiliated to Shanghai Jiao Tong University School of Medicine, Shanghai, China

${ }^{2}$ Institute of Science and Technology for Brain-inspired intelligence, Fudan University, Shanghai, China

Full list of author information is available at the end of the article
}

(c) The Author(s). 2020 Open Access This article is licensed under a Creative Commons Attribution 4.0 International License, which permits use, sharing, adaptation, distribution and reproduction in any medium or format, as long as you give appropriate credit to the original author(s) and the source, provide a link to the Creative Commons licence, and indicate if changes were made. The images or other third party material in this article are included in the article's Creative Commons licence, unless indicated otherwise in a credit line to the material. If material is not included in the article's Creative Commons licence and your intended use is not permitted by statutory regulation or exceeds the permitted use, you will need to obtain permission directly from the copyright holder. To view a copy of this licence, visit http://creativecommons.org/licenses/by/4.0/ The Creative Commons Public Domain Dedication waiver (http://creativecommons.org/publicdomain/zero/1.0/) applies to the data made available in this article, unless otherwise stated in a credit line to the data. 


\section{Background}

Nausea and vomiting during pregnancy (NVP), commonly known as morning sickness, affects as many as $80 \%$ of pregnant women [1]. Typical NVP occurs during the first trimester and is self-limiting, subsiding before 20 weeks of gestation [2]. It is considered to represent a healthy pregnancy, which has been linked to favorable obstetric and neonatal outcomes, including lower risk of miscarriage [3] and reduced odds for low birth weight and preterm birth $[4,5]$.

Clinically, around $15 \%$ of pregnant women continue to have NVP beyond 20 weeks of gestation [1]. Severe prolonged NVP correlates closely with vitamin B deficiency, chronic psychological stress, and weight loss [2]. These conditions play a crucial role in shaping fetal development and subsequent child brain and behavior development [6-9]. One study reported that severe prolonged NVP was associated with increased internalizing and externalizing behaviors in the offspring which reflect mood changes and conflict with others [10] and another reported decreased social responsiveness [11], highlighting the need for a large-scale evaluation of the long-term effects of exposure to severe prolonged NVP. As observed in two recent studies, clinical diagnosis of maternal hyperemesis gravidarum was associated with an increased risk of autism spectrum disorders in the offspring $[12,13]$. Further, there is increasing evidence in general that exposure to adverse circumstances during pregnancy can significantly affect fetal brain development [14-17]. No previous study has examined the biological effects of severe prolonged NVP on brain morphology in children. Moreover, anatomical brain abnormalities play a crucial role in children with neurodevelopmental disorders $[18,19]$. In this context, we performed a large-scale investigation of the association between severe prolonged nausea and vomiting in pregnancy and psychiatric and emotional problems, and brain structure, years later in children. We also assessed which brain differences may mediate the effects of severe prolonged NVP on psychiatric and cognitive problems, to potentially understand better what effects severe prolonged NVP may have.

We hypothesized that prenatal exposure to severe prolonged NVP is associated with poor cognitive performance and a high risk of emotional and psychiatric problems in children and that the underlying mechanisms could be related to structural differences in the brain. To test the hypothesis, the specific aims were as follows: (1) to assess the association between exposure to severe prolonged NVP and cognitive performance, emotional problems, and brain morphology in the children using data from the Adolescent Brain Cognitive Developmental (ABCD) Study [20]; (2) to examine whether brain morphology mediates the association between exposure to severe prolonged NVP and neurodevelopment in the children; and (3) to externally validate the results of severe nausea and vomiting (clinically diagnosed of hyperemesis gravidarum (HG) that requires antenatal hospital admission) and emotional and psychiatric problems, using data from the Danish National Registry System [21-27].

\section{Methods \\ Participants}

The dataset used for this investigation was from the Annual Curated Data Release 2.01 from the ABCD consortium (https://abcdstudy.org/). A total of 10,710 participants aged 9 to 11 years were included in the ABCD study, which was a large national-based longitudinal study that recruited children across 21 research sites across the USA [20]. The $A B C D$ investigators obtained written and oral informed consent from parents and children, respectively [28]. More details of the study subjects and the collection are provided at the ABCD website (https://abcdstudy.org/scientists/protocols/) and also are described elsewhere [20, 29].

\section{Structural neuroimage processing}

We obtained preprocessed structural magnetic resonance imaging (MRI) data (T1 and T2) using the ABCD pipeline, with all the data preprocessing procedures performed by the $A B C D$ team as described in their image processing paper [29]. Briefly, the data preprocessing included the following procedures: (1) T1w and T2w structural images were corrected for gradient nonlinearity distortions; (2) T2w images were registered to T1w images; (3) intensity normalization and B1 inhomogeneity correction; (4) images were rigidly registered and resampled into alignment with a custom, in-house atlas created by the ABCD data preprocessing team for participants of this age; (5) FreeSurfer (version: v5.3.0) was used for cortical surface reconstruction and subcortical segmentation which included skull-stripping, white matter segmentation, initial mesh creation, correction of topological defects, generation of optimal white and pial surfaces, and nonlinear registration to a spherical surface-based atlas based on the alignment of sulcal/ gyral patterns; (6) images were registered to a spherical atlas based on surface-based nonlinear registration, and the cerebral cortex was parcellated into 74 regions per hemisphere [30]; and (7) and finally, morphometric measures included the cortical area, volume, and thickness of each brain region and were used in our analysis.

The quality control of the processed images was done by the ABCD team. The trained staff manually reviewed the accuracy of cortical surface reconstruction, and only the data recommended for use was included in our analysis [29]. A total of 481 participants who failed to pass the quality control of the $\mathrm{ABCD}$ team were removed 
from the subsequent analysis, and there was no difference in the percentage of subjects who failed the quality control between the two groups. In addition, 360 participants who did not contain the full information of both the exposure to NVP and the structural images were excluded. Finally, 10,710 participants remained in this study, and the demographic characteristics of these participants are summarized in Table 1 . All brain measurements and behavioral variables used in this study were collected at the ABCD baseline time when the average age was 119 months.

\section{Maternal nausea and vomiting during pregnancy}

Information about NVP was collected through a structured maternal interview. Mothers in the ABCD study were asked if they had severe NVP extending past the 6th month of gestation, or NVP that was accompanied by weight loss, and were included in the group named SNVP studied here if they answered yes, and if the answer was no, they were in the control group (ABCD question dhx01, item devhx_10a3_p).

\section{Cognition measures}

Cognitive development was assessed with NIH Toolbox (abcd_tbss01) which consists of seven validated and reliable psychometric tests (the Picture Vocabulary Test, the Flanker Inhibitory Control and Attention Test, the List Sorting Working Memory Test, the Dimensional Change Card Sort Test, the Pattern Comparison Processing Speed Test, the Picture Sequences Memory test, and the Oral Reading Recognition Test) [31, 32].

\section{Emotional and psychiatric problems}

Emotional and behavioral problems were assessed by the Child Behavioral Checklist (CBCL), completed by the child's caregiver. CBCL has high test-retest stability and good internal consistency and contains 113 items that measure broad scopes of child behavior across the past 6 months [33]. The resulting syndrome scores including anxious/depressed, withdrawn/depressed, somatic complaints, social problems, thought problems, rulebreaking behavior, and aggressive behavior were derived $[34,35]$. Because this checklist includes some items related to psychiatric problems including depression that are associated with NVP (Additional file 1: Table S1), we refer to these measures in this paper as "emotional and psychiatric problems."

More details of these behavior assessments are provided in Additional file 1: Table S2 [20, 28, 29, 31, 32, 36-39] and are also available at the ABCD website (https://abcdstudy.org/scientists-protocol.html).

\section{Statistical analysis \\ Association analysis}

A linear mixed-effect model (LMM) was used to test the associations of the exposure to NVP with the brain morphometric measures and with the children's cognitive scores from the NIH Cognitive Toolbox and the psychiatric problems scores from the Child Behavior Checklist noted above that are provided by ABCD. As recommended by the ABCD and used in many studies [40, 41], a linear mixed-effects model (LMM) was used to model the correlated observations within families due to twins and siblings and at sites. In this way, the LMM was specified to model family nested within the site. The LMM

Table 1 Demographic characteristics of the 10,710 ABCD participants

\begin{tabular}{|c|c|c|c|c|}
\hline & \multirow[t]{2}{*}{ Controls $(N=9214)$} & \multirow[t]{2}{*}{ SNVP $(N=1496)$} & \multicolumn{2}{|l|}{ Comparison } \\
\hline & & & $t / x^{2}$ statistic & $p$ value \\
\hline Age (months) & $119.06 \pm 7.48$ & $118.71 \pm 7.37$ & 1.704 & 0.088 \\
\hline Gender (male/female) & $4358 / 4856$ & $757 / 739$ & 5.631 & 0.018 \\
\hline BMI & $18.66 \pm 4.08$ & $19.48 \pm 4.58$ & -7.141 & $9.87 \times 10^{-13}$ \\
\hline Parents income & $7.70 \pm 2.42$ & $6.76 \pm 2.95$ & 13.48 & $4.47 \times 10^{-41}$ \\
\hline Parents education & $16.80 \pm 2.65$ & $15.55 \pm 3.07$ & 16.56 & $7.32 \times 10^{-61}$ \\
\hline Puberty & $1.58 \pm 0.48$ & $1.72 \pm 0.55$ & -9.935 & $3.71 \times 10^{-23}$ \\
\hline Race (White/Black/Indian/others) & $7151 / 1718 / 277 / 68$ & $965 / 436 / 67 / 28$ & 120.4 & $5.10 \times 10^{-28}$ \\
\hline Use of tobacco (yes/no) & $8062 / 1152$ & $1260 / 236$ & 11.47 & $7.09 \times 10^{-4}$ \\
\hline Use of alcohol (yes/no) & $6912 / 2302$ & $1186 / 310$ & 13.31 & $2.65 \times 10^{-4}$ \\
\hline Use of marijuana (yes/no) & $8767 / 447$ & $1382 / 114$ & 18.18 & $2.01 \times 10^{-5}$ \\
\hline Use of cocaine/crack (yes/no) & $9179 / 35$ & $1485 / 11$ & 1.556 & 0.212 \\
\hline Use of heroin/morphine (yes/no) & $9204 / 10$ & $1491 / 5$ & 0.903 & 0.342 \\
\hline Use of oxytocin (yes/no) & $9192 / 22$ & $1489 / 7$ & 0.470 & 0.493 \\
\hline
\end{tabular}

The data was shown as mean \pm standard deviation 
Table 2 Characteristics of the Danish study population born between 1978 and 2012 at birth according to maternal hyperemesis gravidarum status $(N=2,092,897)$

\begin{tabular}{lll}
\hline & \multicolumn{3}{l}{ Maternal hyperemesis } & gravidarum status \\
\cline { 2 - 3 } & Yes & No \\
& $(N=21,282)$ & $(N=2,071,615)$ \\
Characteristics & $N(\%)$ & $N(\%)$ \\
\hline
\end{tabular}

\begin{tabular}{lrr}
\hline Sex & & \\
Boys & $9702(45.6)$ & $1,064,828(51.4)$ \\
Girls & $11,580(54.4)$ & $1,006,787(48.6)$
\end{tabular}

Preterm

No

Yes

Missing

Low birth weight

No

Yes

Missing

Parity

1

2

$\geq 3$

Maternal age (years)

$\leq 25$

26-30

31-35

$>36$

Paternal age (years)

$\leq 25$

26-30

31-35

$>36$

Missing

Maternal education level

$$
\text { 0-9 }
$$

10-14

$\geq 15$

Missing

Maternal psychiatric disorders

$$
\begin{aligned}
& \text { No } \\
& \text { Yes }
\end{aligned}
$$

Paternal psychiatric disorders

No

Yes

Maternal original

Born in Denmark

Not born in Denmark

Missing

$\begin{array}{cc}19,573(92.0) & 1,899,027(91.7) \\ 1088(5.1) & 95,375(4.6) \\ 621(2.9) & 77,213(3.7)\end{array}$

$20,146(94.6)$

$840(4.0)$

296 (1.4)

9488 (44.6)

7916 (37.2)

3878 (18.2)

7112 (33.4)

7619 (35.8)

4868 (22.9)

$1683(7.9)$

3608 (17.0)

6683 (31.4)

5993 (28.2)

4393 (20.6)

605 (2.8)

7026 (33.0)

9257 (43.5)

4454 (20.9)

545 (2.6)

19,042 (89.5)

$2240(10.5)$

19,991 (93.9)

1291 (6.1)

$16,103(75.7)$
$5151(24.2)$
$28(0.1)$
$1,961,425(94.7)$

80,443 (3.9)

29,747 (1.4)

922,209 (44.5)

770,725 (37.2)

378,681 (18.3)

569,002 (27.5)

785,543 (37.9)

526,372 (25.4)

190,698 (9.2)

293,050 (14.1)

659,191 (31.8)

616,331 (29.8)

413,205 (20.0)

$89,838(4.3)$

577,435 (27.9)

905,428 (43.7)

555,847 (26.8)

32,905 (1.6)

$1,964,722$ (94.8)

106,893 (5.2)

$1,981,118(95.6)$

90,497 (4.4)

$1,853,108$ (89.5)

$4681(0.2)$
213,826 (10.3)
Table 2 Characteristics of the Danish study population born between 1978 and 2012 at birth according to maternal hyperemesis gravidarum status ( $N=2,092,897$ ) (Continued)

\begin{tabular}{llc}
\hline & \multicolumn{2}{l}{ Maternal hyperemesis gravidarum status } \\
\cline { 2 - 3 } & $\begin{array}{l}\text { Yes } \\
(N=21,282)\end{array}$ & No \\
& $N(\%)$ & $N(\%)$ \\
Characteristics & & \\
\hline Maternal cohabitation status & $12,590(59.2)$ & $1,158,257(55.9)$ \\
Yes & $8688(40.8)$ & $911,630(44.0)$ \\
No & $4(0.0)$ & $1728(0.1)$ \\
Missing & & \\
\hline
\end{tabular}

was implemented using the MATLAB function fitlme. A morphometry measurement or behavioral score was modeled as the dependent variable, and the exposure to SNVP and the nuisance covariates were modeled as fixed effects, while the family structures nested within sites were modeled as random effects. The following variables were used as nuisance covariates of no interest: children's age, sex, body mass index, puberty score, race (coded as 3-column dummy variables), and parents' income, number of years of education, use of tobacco, use of alcohol, use of marijuana, use of cocaine/crack, use of heroin/morphine, and use of oxytocin during pregnancy, in line with previous studies [40, 42, 43]. A $t$-statistic and Cohen's $d$ were calculated based on the coefficient of interest variable (the exposure to SNVP) in the linear mixed-effect model to reflect the association between the exposure to SNVP and the dependent variable. A positive $t$ value means that the dependent variable is higher in the SNVP exposure group. Finally, false discovery rate (FDR) and Bonferroni corrections to correct the results for multiple comparisons were performed.

\section{Mediation analysis}

A standard mediation analysis was performed using the Mediation Toolbox developed by Tor Wager's group (https:/github.com/canlab/MediationToolbox), which has been widely used in neuroimaging studies [44, 45]. A standard 3-variable path model was used here [46], with the detailed methodology described in the supplementary material of [44]. Briefly, mediation analysis tests whether the covariance between two variables can be explained by the third variable (the mediator). The two hypotheses investigated the relation of cortical morphometry with psychiatric and cognitive problems and exposure to SNVP. For the first hypothesis, the independent (predictor) variable was the exposure to SNVP and the dependent (predicted) variable was the psychiatric problems total score. The proposed mediator (in the indirect path) was a morphometric measure. For the second hypothesis, the independent (predictor) variable was the exposure to SNVP 
and the dependent (predicted) variable was the Cognition Total Composite Score. The proposed mediator was a morphometric measure. Confounding variables as in the association analysis were regressed out in the mediation model. The significance of the mediation was estimated by the bias-corrected bootstrap approach (with 10,000 random samplings).

\section{External validation using the Danish nationwide cohort study}

All the Danish data are obtained from national registers [22-27] for which detailed information is presented in Additional file 1: Table S3. All residents have a unique personal identification number that permits an accurate linkage of individual-level data from registers [21]. We conducted a population-based cohort study including all singleton live births born in Denmark during 1978-2012 $(N=2,100,158)$. We excluded 463 children who had errors in gestational age $(<154$ or $>315$ days $)$ and 6798 children without links to their fathers. The final analyses included a total of 2,092,897 children (Additional file 1: Figure S1). We followed them from birth until the date of the first diagnosis of a psychiatric disorder, emigration, death, 18-year birthday, or 31 December 2016, whichever came first. From 1969 to 1993, the diagnostic system used was the Danish modification of the International Classification of Disease, 8th revision (ICD-8), and from 1994 the International Classification of Disease, 10th revision (ICD-10).

Information on hyperemesis gravidarum (HG) was obtained from the Danish National Patient Register (DNPR). Diagnoses for HG are defined as $I C D-8$ code: 638 and $I C D-10$ code: O21 [47].

Clinical diagnoses for psychiatric disorders were identified from the DNPR and the Danish Psychiatric Central Research Register (DPCRR) [22, 23]. In order to focus on neuropsychiatric disorders with onset usually occurring in childhood and adolescence, and also to be consistent with previous publications based on Danish registers $[48,49]$, we categorized them into the following groups: pervasive developmental disorders including childhood autism; developmental disorders including language, learning, and motor skills disorders; and behavioral and emotional disorders with onset usually occurring in childhood and adolescence, including attentional-deficit/hyperactivity disorders, conduct disorders/oppositional defiant disorders, and emotional disorders. The diagnostic codes for ICD-8 and ICD-10 are provided in Additional file 1: Table S4. Based on previous studies using information from the Danish national registers, diagnoses for $\mathrm{ADHD}$ are defined according to ICD-8 code: 30801 and ICD-10 codes: F90 and F98.8. ADHD case was also defined when the individual had at least two redeemed prescriptions for ADHD-specific medication from the National Prescription Register [48]. The Anatomical Therapeutic Chemical codes for ADHD-specific medication were N06BA04 (methylphenidate) and N06BA09 (atomoxetine). Children who had redeemed N06BA07 (modafinil) were included as ADHD cases only if they had previously redeemed a prescription for either N06BA04 or N06BA09 [50].

The association of exposure to $\mathrm{HG}$ and behavioral and psychiatric problems in the offspring was estimated by the Cox proportional hazard model, with a child's age as the time scale. Evaluation of log-minus-log survival curves showed that the curves were roughly parallel (Additional file 1: Figure S2). Covariates were identified a priori as potential confounding factors and/or risk factors for behavioral and emotional problems in children and adolescents. In the final model, the following potential confounders were included: sex of the child, calendar year of birth, maternal country of origin, maternal education and cohabitation status, parental age, and parental psychiatric disorder history before childbirth $[48,50]$. To take account of the ICD code change, we restricted analyses to offspring born after 1994 when ICD-10 codes were used.

\section{Role of the funding source}

The funding sources had no role in the study design; in the collection, analysis, and interpretation of the data; in the writing of the report; and in the decision to submit the paper for publication.

\section{Results}

Severe prolonged NVP is associated with psychiatric and cognitive problems in children aged 9-11 years

All the psychiatric problems scores (abcd_cbcls01) were significantly higher in the group with SNVP with $t$ values ranging from 5.10 to 9.09 (Bonferroni corrected all $p<1 \times 10^{-5}$, Additional file 1: Table S1, Fig. 1b, c). The total psychiatric problems score was 22.7 in the SNVP group and 17.0 in the controls $\left(p<10^{-10}\right.$, Additional file 1: Table S1), indicating that the children exposed to SNVP had a total psychiatric problems score that was $25.2 \%$ higher on average. The psychiatric problems associated with SNVP included ADHD, depression, and social problems (Additional file 1: Table S1).

Six out of ten cognitive scores (abcd_tbss01) were significantly lower in the group whose mother had severe prolonged nausea and vomiting during pregnancy (FDR correction, $p<0.05$, Fig. 1a and Additional file 1: Table $\mathrm{S} 1)$. The total cognitive performance score was 83.7 in the SNVP group and 86.9 in the controls $\left(p=1.4 \times 10^{-5}\right.$, Additional file 1: Table S1), indicating that the children exposed to SNVP had a total cognitive performance score that was $3.7 \%$ lower on average. 
Severe prolonged NVP is associated with low volume and area of some cortical regions

The total cortical volume and area were significantly lower in the children with exposure to SNVP with $t$ value -4.02 (Cohen's $\left.d=-0.08, p=6.0 \times 10^{-5}\right)$ and $-3.51(d=-0.07$, $p=4.4 \times 10^{-4}$ ) respectively. The reductions of volume were especially of the anterior and posterior cingulate cortex, precuneus, superior and middle frontal gyrus and superior medial prefrontal cortex, the dorsolateral prefrontal cortex, temporal pole, calcarine cortex, and post- and precentral gyrus (FDR corrected, $p<0.05$, Fig. 2a, Additional file 1: Table S5). Similar brain areas, including the anterior and posterior cingulate cortex, precuneus, superior medial prefrontal cortex, inferior temporal cortex, insula, and postcentral cortex, showed significantly lower cortical areas in the children with exposure to NVP (FDR corrected, $p<0.05$, Fig. 2b, Additional file 1: Table S5). No brain regions showed increased cortical volume and area in the SNVP group. No brain regions showed altered cortical thickness in the children with prenatal exposure to SNVP after FDR correction $(p<0.05)$.

The lower cortical volume and area of some brain regions significantly mediate the association between exposure to SNVP and psychiatric and cognitive problems

The indirect effect of the exposure to SNVP on the psychiatric problems was significantly mediated by the mean cortical volume of the significant brain regions shown in Fig. 2a (3.7\% of the total effect size measured by the variance explained (VE), $p=2.0 \times 10^{-5}, \beta=0.176$, $95 \%$ CI, 0.105 to 0.271 , Fig. 3a) and by the mean cortical area of the significant brain regions shown in Fig. 2a $\left(\mathrm{VE}=3.6 \%, p=3.1 \times 10^{-5}, \beta=0.172,95 \% \mathrm{CI}, 0.101\right.$ to 0.265 , Fig. $3 \mathrm{~b})$. The mediation results for each brain area are shown in Additional file 1: Table S6, with the areas involved including the anterior cingulate cortex, superior and middle frontal gyrus, precuneus, and temporal pole (FDR correction, $p<0.05$ ).

The indirect effect of the exposure to SNVP on cognitive performance was significantly mediated by the mean cortical volume of the significant brain regions shown in Fig. $2 \mathrm{~b}\left(\mathrm{VE}=13.3 \%, p=8.5 \times 10^{-7}, \beta=-0.122,95 \% \mathrm{CI}\right.$, -0.174 to -0.077 , Fig. 3c). The indirect effect of the exposure to SNVP on cognitive performance mediated by the mean cortical area of the significant brain regions shown in Fig. 2b was also significant (VE $=14.9 \%, p=$ $8.0 \times 10^{-7}, \quad \beta=-0.136,95 \% \mathrm{CI},-0.192$ to -0.088 , Fig. 3d). The mediation results for each brain area are shown in Additional file 1: Table S7, with the areas involved including the anterior and posterior cingulate cortex, superior and middle frontal gyrus, precuneus, inferior temporal cortex, and insula (FDR correction, $p<$ 0.05).

\section{External validation results using the Danish nationwide} cohort study

In the Danish National Cohort, 21,282 (1.0\%) children were born to mothers with hyperemesis gravidarum. The proportion of offspring born to mothers with HG increased over time (Additional file 1: Figure S3). Table 2 presents the baseline characteristics of the participants. Compared with unexposed offspring, exposed offspring were more likely to be born to younger mothers. Compared with mothers who did not experience HG, mothers with HG were more likely to have a lower level of education and more comorbid psychiatric disorders. Table 3 shows that compared to unexposed children, children of mothers with HG had increased risks of behavioral and emotional disorders (HR, 1.24; 95\% CI, 1.16-1.33), attention-deficit/hyperactivity disorders (HR, 1.22; 95\% CI, 1.12-1.33), conduct disorders/oppositional defiant disorders (HR, 1.31; 95\% CI, 1.02-1.67), emotional disorders ( $\mathrm{HR}, 1.40 ; 95 \% \mathrm{CI}, 1.11-1.77)$, pervasive developmental disorders (HR, 1.18; 95\% CI, 1.05-1.34), childhood autism (HR, 1.28; 95\% CI, 1.06-1.56), and developmental disorders (HR, 1.36; 95\% CI, 1.09-1.70). The results remained unchanged when restricting analyses to offspring born after 1994 (Additional file 1: Table S8).

\section{Discussion}

In this large, nationally representative cohort of 10,710 US children, our findings provide the first evidence that prenatal exposure to prolonged severe nausea and vomiting in pregnancy (SNVP) is associated with a range of psychiatric problems including ADHD symptoms, and with poor cognitive performance. External validation from the Danish National Cohort also suggested that severe nausea and vomiting in pregnancy is associated with psychiatric problems (including ADHD), and behavioral and emotional problems. The findings also show that children with prenatal exposure to SNVP have significantly reduced total cortical volume and area; and that the particular brain areas with significant reductions include the anterior and posterior cingulate cortex, the precuneus, the superior medial prefrontal cortex, the superior and middle frontal gyri, the temporal lobe, the insula, the calcarine cortex, and the postcentral gyrus. Further mediation analyses showed that the reduced volume or area of many of these brain regions significantly mediates the effects of SNVP on psychiatric problems and on cognition. These findings provide a unique contribution demonstrating that prenatal exposure to prolonged and severe NVP is associated with psychiatric problems and poor cognitive performance in the offspring and that the effects are mediated via the altered brain morphology. The findings highlight the clinical 


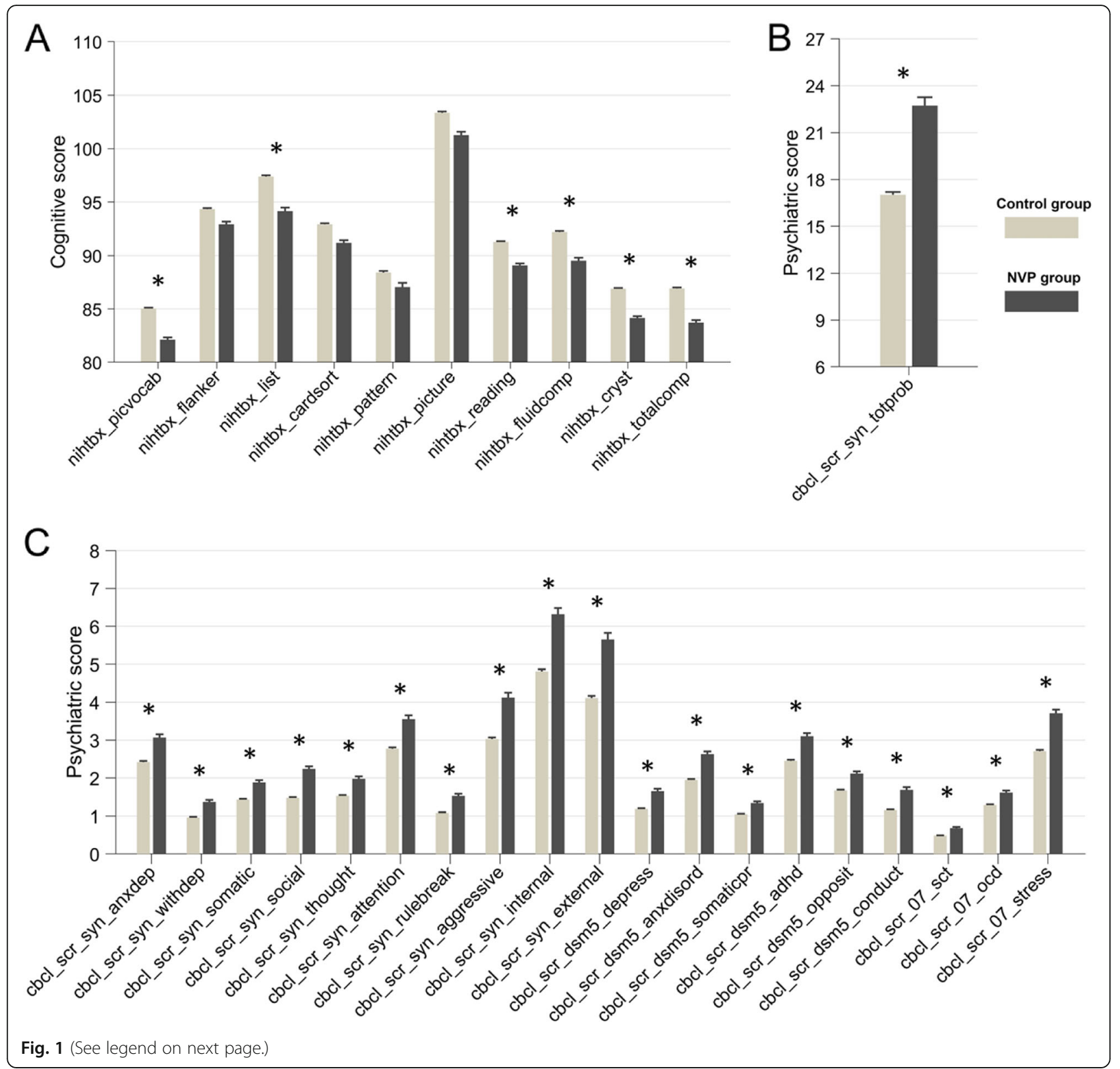




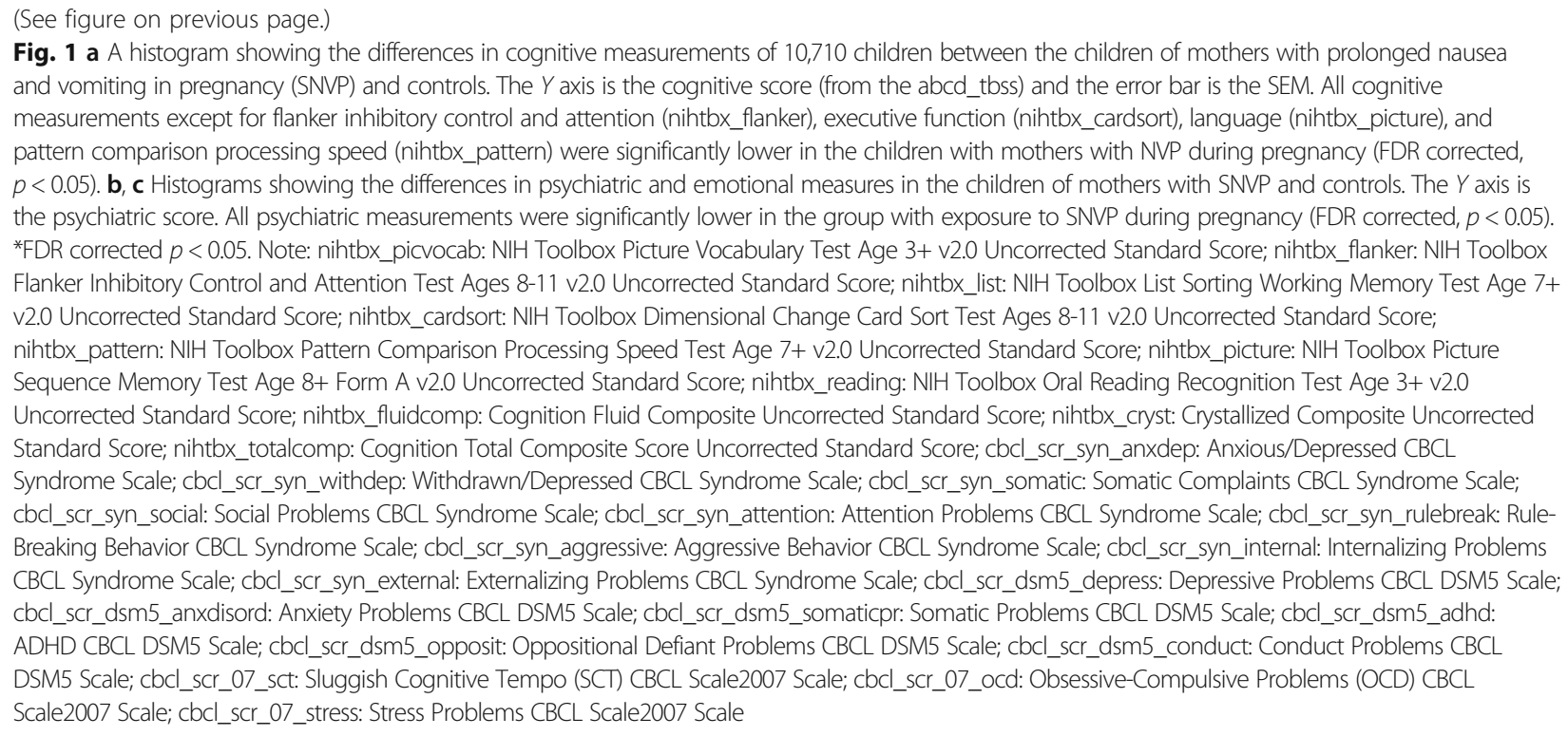

importance of attention to prolonged nausea and vomiting in pregnant women for mental health in the offspring.

Previous studies on the association of exposure to severe prolonged NVP and the risk of adverse health outcomes in the offspring are scarce. Two are mentioned in the introduction [10,11], and these much smaller studies ( $n=560$ and 287, respectively) reported some changes in cognition and emotional and behavioral problems. The present study was much more powerful than previous studies, with a very large sample $(n=10$, 710) demonstrating that a wide range of psychiatric (Table S2) and cognitive measures (Table S2) are associated with severe prolonged NVP and with externalvalidation of the major psychiatric findings in an independent national sample $(n=2,092,897$, Table 3$)$.

No previous study has examined the association between exposure to severe prolonged NVP and brain morphology in the offspring. We found that exposure to SNVP is associated with reduced cortical areas to the entire brain surface. Studies in humans and primates have indicated that early brain development is characterized by an initial expansion of the cortical surface areas [51-53]. During the early stage of development, the expansion of the cortical areas may be particularly susceptible to environmental insults [54]. Accumulating evidence suggested that fetal exposure to adverse intrauterine exposures, i.e., cocaine, alcohol, cigarettes, or air pollution, is associated with reduced cortical areas [55-59]. For example, two studies have reported that fetal exposure to maternal psychosocial stress (depression/anxiety) during the second trimester of pregnancy is associated with decreased cortical thickness/volume [17, 60].
The brain areas that we have found to be significantly related to SNVP include anterior and posterior cingulate gyrus, precuneus, temporal pole, inferior temporal cortex, prefrontal areas including the superior and middle frontal gyri, and the superior medial prefrontal cortex, and pre- and postcentral gyri. The anterior cingulate cortex is involved in emotion and relating actions to rewards and punishers and is implicated in psychiatric disorders including ADHD and depression [61-63]. A previous case-control study demonstrated that individuals with ADHD had a smaller total cortical volume (7.3\%), and surface area (4.3\%), compared to controls, and the reduced surface area in the precuneus may be a major driver of the volume differences [64]. In the present investigation, the mediation analyses showed that reduced cortical area in the precuneus partly mediated the association of exposure to SNVP and emotional and psychiatric problems in offspring. The precuneus is a notable region for its role in awareness, episodic memory, and visuospatial processing, suggesting the potential relevance of this region for ADHD-related symptoms [65], for autism [66] and depression [67], and the precuneus is closely connected with the posterior cingulate cortex [61]. Prefrontal cortical areas are also linked to ADHD [68]. Our results from the Danish national population study confirmed that maternal HG is associated with a $44 \%$ increased risk of ADHD in the offspring.

These brain areas with regions significantly related to SNVP have also been consistently linked with cognitive functions [59, 69]. The superior and middle frontal gyri are involved in cognitive execution networks, such as reasoning, planning, and language comprehension [59]. The superior medial prefrontal cortex and frontal pole are also involved in cognitive functions, including 

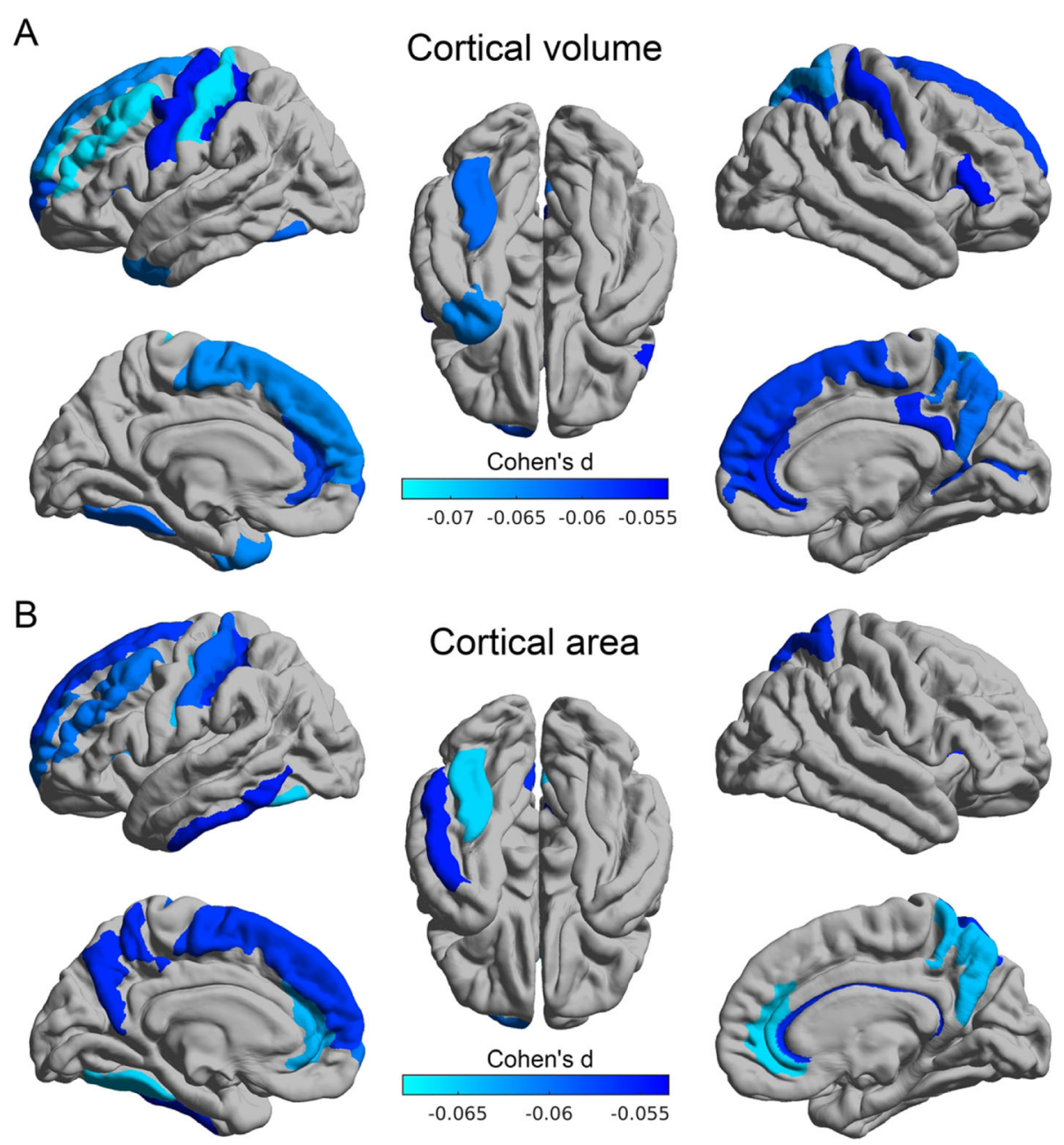

Fig. 2 a Brain regions with their cortical volume significantly lower in children whose mothers had SNVP during pregnancy (FDR corrected, $p<0.05$ ). b Brain regions with their cortical area significantly lower in the children whose mothers had SNVP during pregnancy (FDR corrected, $p<0.05$ )

planning [70]. Therefore, the reductions in these brain areas may help to account for the observed significant associations between SNVP and lower cognitive performance in the offspring.

There are many changes in the brain structure during development, including in the area, volume, and thickness of different cortical areas, and such differences are found in disorders like attention-deficit/hyperactivity disorder and autism spectrum disorder and in other childhood adversities [71-73]. Differences in these measures may reflect, for example, the extent of neuronal dendrites in a cortical area, and the number of synapses on each neuron. Studies with large populations are needed for reliable results. A key feature of the current investigation is that the morphometric effects were measured in 10,710 children, a study sample larger than that in most previous studies [73]. In terms of the interpretation of the reduced volumes and areas reported here associated with SNVP, we have reported in another study of associations involving maternal age at childbirth of the 10,710 participants, in which there was a linear relationship between the reduction in total cortical volume and area in children, and their behavioral and cognitive problems of the type described here [74].

\section{Possible mechanisms}

The exact mechanism underlying the association between prenatal exposure to prolonged severe NVP and neurodevelopmental problems, including differences in the brain structure and psychiatric and cognitive problems, are not closely understood. First, it is plausible that altered brain morphology may be a possible mechanism via which exposure to severe prolonged NVP is associated with increased psychiatric, emotional, and cognitive problems. Second, severe prolonged NVP may indicate an environmental insult during pregnancy since severe prolonged NVP correlates closely with greater maternal stress [75]. Maternal elevated levels of stress during 


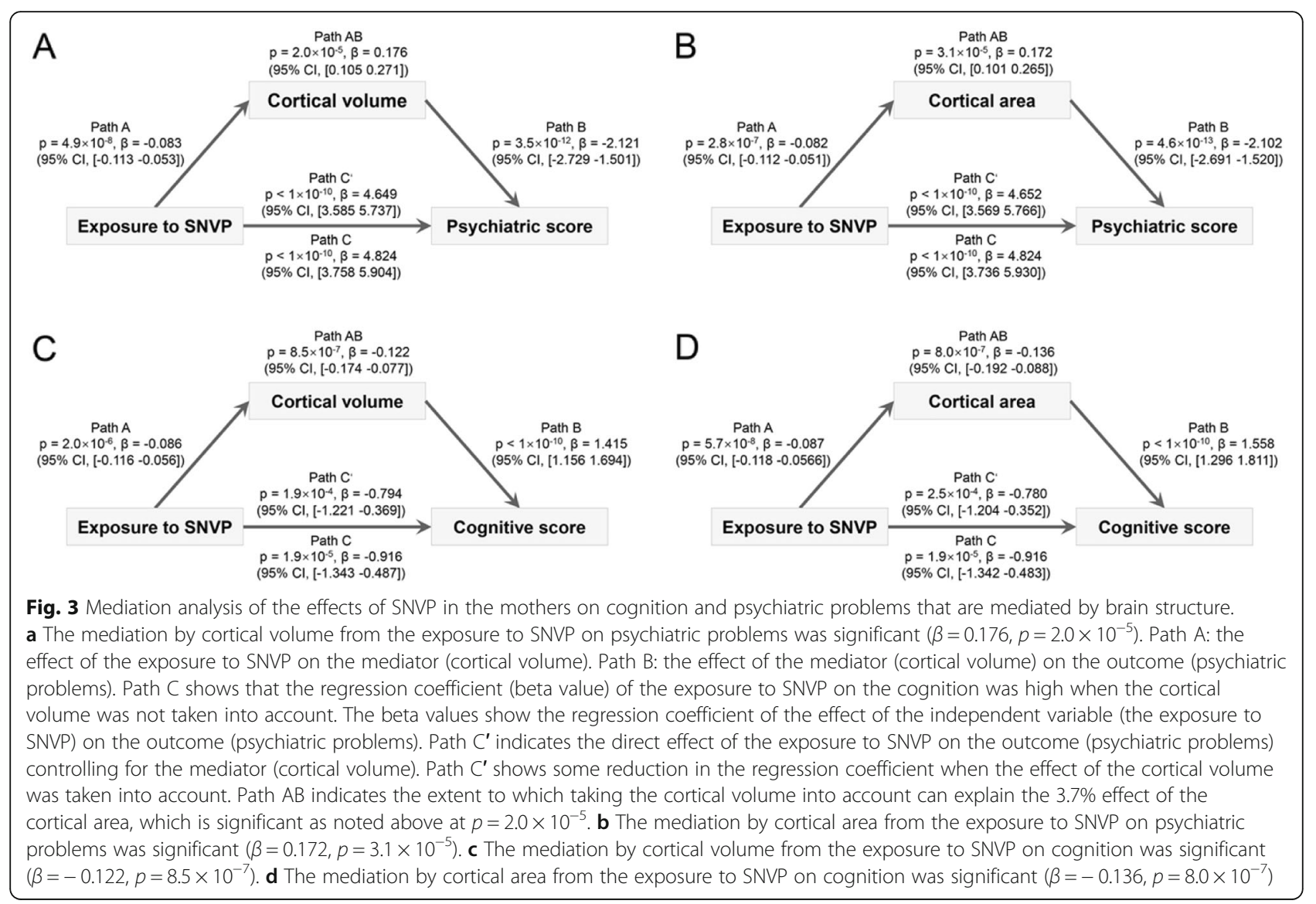

pregnancy could increase the release of placental corticotrophin-releasing hormone $(\mathrm{pCRH})$, which could be produced from the placenta as early as the eighth week of gestation and increase exponentially across the gestation stage [76]. Notably, pCRH synthesis increase is responsive to maternal stress signals [76]. pCRH represents an integrative pathway through which diverse prenatal environmental insults inform the fetus of the condition and shape the fetal developmental trajectories [77]. Considerable evidence indicates that fetal exposure to excessive pCRH concentrations is associated with behavioral consequences during infancy and neuropsychiatric outcomes in childhood [78, 79]. Another plausible interpretation is that maternal stress induced by HG could result in elevated maternal cortisol levels during pregnancy [12]. Because cortisol can pass through the immature blood-brain barrier and targets glucocorticoid receptors, fetal exposure to elevated cortisol is associated with increased concentrations of glucocorticoids, which may play a critical role in modulating normal brain development $[80,81]$. This hypothesis is also supported by animal models demonstrating that exposure to prenatal maternal stress is associated with changes in brain morphometry [82].

\section{Strengths and limitations}

Our study had several strengths. First, the very large sample sizes facilitate generalization of the findings to other populations. Second, because comprehensive data is available in the ABCD study, we were able to control for a variety of potential confounding factors. Third, the participants in the current study were preadolescent children, so the morphological findings were unlikely to be confounded by smoking or substance use. The results described show that there is an important association between prolonged severe nausea and vomiting in pregnancy and psychiatric and cognitive problems in children, which are of clinical relevance, suggesting that interventions to limit the prolonged severe nausea and vomiting in pregnancy may be important.

Our study also has some limitations. First, maternal NVP was obtained through self-reports which might be subject to measurement error as in any observational study; however, if present, any misclassification should be non-differential with respect to the outcome measures and would thus have underestimated the true association. Additionally, the prevalence of prolonged severe NVP in our study is $13.98 \%$; this is comparable to the prevalence of $10-15 \%$ reported in the general population. Nevertheless, in the external validation study, 
Table 3 Incidence rate and hazard ratio of specific psychiatric disorders in offspring born during 1995-2012 in Denmark relating to maternal hyperemesis gravidarum

\begin{tabular}{|c|c|c|c|c|}
\hline & No. of cases, $N$ & Incidence rate, rate per 1000 person-years & Model 1, HR (95\% Cl) & Model 2, HR (95\% Cl) \\
\hline \multicolumn{5}{|c|}{ Behavioral and emotional disorders* } \\
\hline No HG & 41,869 & 3.07 & Ref & Ref \\
\hline Maternal HG & 595 & 3.67 & $1.27(1.17-1.38)$ & $1.20(1.10-1.30)$ \\
\hline \multicolumn{5}{|c|}{ Attention-deficit/hyperactivity disorders } \\
\hline No HG & 32,177 & 2.57 & Ref & Ref \\
\hline Maternal HG & 437 & 2.85 & $1.23(1.12-1.35)$ & $1.16(1.06-1.28)$ \\
\hline \multicolumn{5}{|c|}{ Conduct disorders/oppositional defiant disorders } \\
\hline No HG & 2024 & 0.15 & Ref & Ref \\
\hline Maternal HG & 29 & 0.18 & $1.31(0.91-1.89)$ & $1.06(1.71-1.57)$ \\
\hline \multicolumn{5}{|c|}{ Emotional disorders } \\
\hline No HG & 2884 & 0.21 & Ref & Ref \\
\hline Maternal HG & 44 & 0.27 & $1.34(1.00-1.81)$ & $1.33(0.98-1.89)$ \\
\hline \multicolumn{5}{|c|}{ Pervasive developmental disorders } \\
\hline No HG & 17,478 & 1.31 & & \\
\hline Maternal HG & 232 & 1.46 & $1.23(1.08-1.40)$ & $1.19(1.05-1.36)$ \\
\hline \multicolumn{5}{|l|}{ Childhood autism } \\
\hline No HG & 6877 & 0.50 & Ref & Ref \\
\hline Maternal HG & 100 & 0.61 & $1.32(1.08-1.61)$ & $1.19(0.97-1.45)$ \\
\hline \multicolumn{5}{|c|}{ Developmental disorders ${ }^{\#}$} \\
\hline No HG & 3362 & 0.24 & Ref & Ref \\
\hline Maternal HG & 55 & 0.33 & $1.43(1.10-1.86)$ & $1.33(1.02-1.75)$ \\
\hline
\end{tabular}

HG hyperemesis gravidarum, HR Hazard ratio, Model 1 children's age at time scale, Model 2 children's age at time scale, sex, year of birth, parity, parental age at birth, maternal education level, maternal country of origin, maternal cohabitation, and parental psychiatry disorders

*Behavioral and emotional disorders with onset usually occurring in childhood and adolescence

\#Developmental disorders including language, learning, and motor skills disorders

SNVP was ascertained according to clinical diagnosis, and the findings were similar. Second, we do not have treatment data in the ABCD dataset for SNVP. Treatment of NVP may be a marker of greater severity [83], which may magnify the strength of the association. Third, the use of tobacco, alcohol, and marijuana was higher in the SNVP group, and although we controlled for these factors in all analyses, the exposed and unexposed group may differ in some characteristics that were not measured, and thus, residual confounding cannot be ruled out. Fourth, our findings are preliminary. In the current analysis, cognitive performance, behavioral and emotional problems, and brain morphology were measured concurrently. Further studies with assessment at multiple time points to track neuropsychiatric development are warranted to clarify how these relationships change over time.

In Denmark, the comprehensive administrative databases allowed us to access maternal hyperemesis gravidarum that was obtained in an objective manner, which could reduce the chance of recall bias [26]. Moreover, the Danish registers have diagnostic information on mental disorders for the whole population, and all treatments will be provided through the government health care system free of charge to all residents [84]. Nevertheless, the register-based study could not capture individuals who do not seek treatment [23]. Although we adjusted for a number of potential confounders, we did not have information on several other factors such as maternal alcohol consumption. Therefore, the strengths and weaknesses of the Danish study contrast with and complement those of the ABCD cohort. Furthermore, even if these two cohorts have different, unrelated sources of bias, the results are comparable, suggesting that the associations observed in this study are unlikely to be explained by unmeasured confounders [85].

\section{Conclusions}

Our results show for the first time that prolonged severe NVP is associated with robust differences in the brain structure which mediate psychiatric and emotional problems and reduced cognitive performance in the offspring during childhood. The findings highlight the importance of attention to pregnant women with severe prolonged 
NVP, of the need for investigations of whether interventions to limit severe prolonged NVP are beneficial, and of the relevance in considering the prenatal exposure to severe prolonged NVP as a risk factor in the clinical assessment of children with mental health disorders. The fact that these brain differences and the associated psychiatric and cognitive problems were present as long after birth as 9-11 years is an indication that these differences may be relevant through to adulthood. These findings highlight the clinical importance and potential benefits of treatment for prolonged nausea and vomiting in pregnant women, and early screening of the offspring, which could help to reduce the risk of psychiatric disorder in the next generation.

\section{Supplementary information}

Supplementary information accompanies this paper at https://doi.org/10. 1186/s12916-020-01701-y.

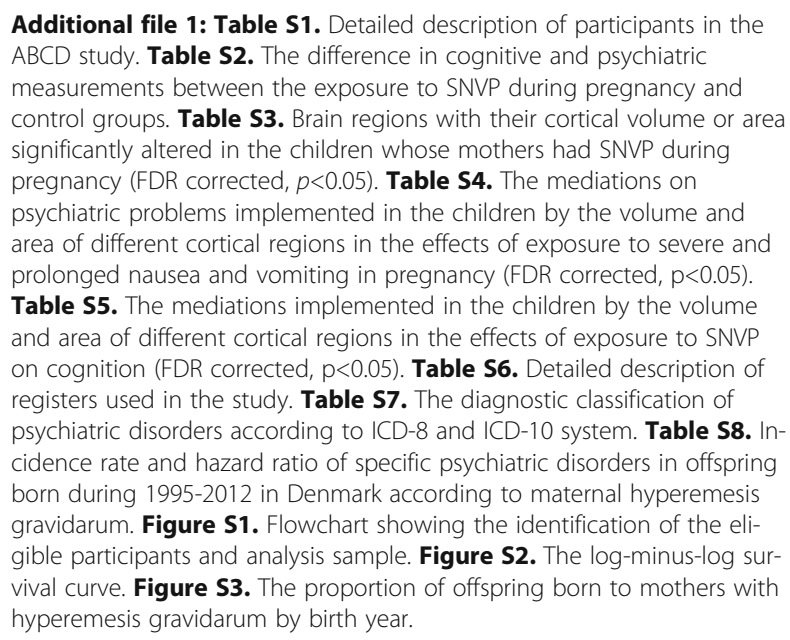

\section{Abbreviations}

NVP: Nausea and vomiting during pregnancy; ABCD: Adolescent Brain Cognitive Developmental; HG: Hyperemesis gravidarum; CBCL: Child Behavioral Checklist; ADHD: Attention-deficit/hyperactivity disorders

\section{Acknowledgements}

Data used in the preparation of this article were obtained from the Adolescent Brain Cognitive Development (ABCD) Study (https://abcdstudy. org), held in the NIMH Data Archive (NDA). This is a multisite, longitudinal study designed to recruit more than 10,000 children age 9-10 and follow them over 10 years into early adulthood. The ABCD Study is supported by the National Institutes of Health and additional federal partners under award numbers U01DA041048, U01DA050989, U01DA051016, U01DA041022, U01DA051018, U01DA051037, U01DA050987, U01DA041174, U01DA041106, U01DA041117, U01DA041028, U01DA041134, U01DA050988, U01DA051039, U01DA041156, U01DA041025, U01DA041120, U01DA051038, U01DA041148, U01DA041093, U01DA041089. A full list of supporters is available at https:// abcdstudy.org/federal-partners.html. A listing of participating sites and a complete listing of the study investigators can be found at https:// abcdstudy.org/scientists/workgroups/. ABCD consortium investigators designed and implemented the study and/or provided data but did not necessarily participate in analysis or writing of this report. This manuscript reflects the views of the authors and may not reflect the opinions or views of the $\mathrm{NIH}$ or $\mathrm{ABCD}$ consortium investigators.
}

\section{Authors' contributions}

HW preformed the literature review, conducted data analysis, and drafted the manuscript. ER, XD, JD, DY, JL, and JF contributed to the interpretation of the data, critically revised the paper, and approved the final version. FL and WC contributed equally to the correspondence work. They developed the study conception, directed the analytic strategy of the study, and supervised the drafting of the manuscript. All authors approved the final manuscript as submitted and agree to be accountable for all aspects of the work.

\section{Funding}

J.Feng is supported by the Shanghai Science and Technology Innovation Plan (No. 15JC1400101 and No. 16JC1420402), the National Natural Science Foundation of China (No. 71661167002 and No. 91630314), the 111 Project (No.B18015), and Shanghai Municipal Science and Technology Major Project (No.2018SHZDZX01). W. Cheng is supported by grants from the National Natural Sciences Foundation of China (No.81701773, 11771010). W. Cheng is also sponsored by the Natural Science Foundation of Shanghai (No. 18ZR1404400). F. Li was supported by funding from the National Natural Science Foundation of China (No. 81761128035, No. 81930095, No.81701334, No. 81703249), Shanghai Municipal Commission of Health and Family Planning (No.2017ZZ02026, No. 2018BR33, No.2017EKHWYX-02), Shanghai Shenkang Hospital Development Center (No.16CR2025B), Shanghai Committee of Science and Technology (No.17XD1403200, No. 19410713500, No.18DZ2313505), Shanghai Municipal Science and Technology Major Project (No. 2018SHZDZX01), Guangdong Key Project in "Development of new tools for diagnosis and treatment of Autism" (2018B030335001), Collaborative Innovation Program of Shanghai Municipal Health Commission (2020CXJQ01), National Human Genetic Resources Sharing Service Platform (2005DKA21300), and Xinhua Hospital of Shanghai Jiao Tong University School of Medicine (2018YJRC03). H. Wang is sponsored by the Natural Science Foundation of Shanghai (No. 81703237). F. Li and H. Wang are also sponsored by the Collaborative Innovation Program of Shanghai Municipal Health Commission (2020CXJQ01). No funding sources contributed to the analysis and interpretation of data nor the writing of this manuscript.

\section{Availability of data and materials}

The datasets used and/or analyzed during the current study are available from the corresponding author on reasonable request.

\section{Ethics approval and consent to participate}

In the Danish national study, the study was approved by the Data Protection Agency (record number 2013-41-2569). By Danish law, no informed consent is required for a register-based study of anonymized data. In the ABCD study, all procedures were approved by a central Institutional Review Board (IRB) at the University of California, San Diego, and in some cases by individual site IRBs (e.g., Washington University in St. Louis) (https://www.sciencedirect.com/ science/article/pii/S1878929317300622). Parents or guardians provided written informed consent after the procedures had been fully explained and children assented before participation in the study.

\section{Consent for publication}

Not applicable.

\section{Competing interests}

The authors declare that they have no competing interests.

\section{Author details}

${ }^{1}$ Department of Developmental and Behavioral Pediatric \& Child Primary Care/MOE-Shanghai Key Laboratory of Children's Environmental Health, Xin Hua Hospital Affiliated to Shanghai Jiao Tong University School of Medicine, Shanghai, China. ${ }^{2}$ Institute of Science and Technology for Brain-inspired intelligence, Fudan University, Shanghai, China. ${ }^{3}$ Key Laboratory of Computational Neuroscience and Brain-Inspired Intelligence, Ministry of Education, Fudan University, Shanghai, China. ${ }^{4}$ Department of Computer Science, University of Warwick, Coventry CV4 7AL, UK. ${ }^{5}$ Oxford Centre for Computational Neuroscience, Oxford OX1 4BH, UK. ${ }^{6}$ Department of Clinical Epidemiology, Aarhus University Hospital, Aarhus N, Denmark. 
Received: 23 March 2020 Accepted: 8 July 2020

Published online: 01 September 2020

\section{References}

1. Lacroix R, Eason E, Melzack R. Nausea and vomiting during pregnancy: a prospective study of its frequency, intensity, and patterns of change. Am J Obstet Gynecol. 2000;182(4):931-7.

2. Niebyl JR. Nausea and vomiting in pregnancy. N Engl J Med. 2010;363(16): 1544-50.

3. Hinkle SN, Mumford SL, Grantz KL, Silver RM, Mitchell EM, Sjaarda LA, Radin RG, Perkins NJ, Galai N, Schisterman EF. Association of nausea and vomiting during pregnancy with pregnancy loss: a secondary analysis of a randomized clinical trial. JAMA Intern Med. 2016:176(11):1621-7.

4. Chortatos A, Haugen M, Iversen PO, Vikanes $\AA$, Eberhard-Gran M, Bjelland EK, Magnus P, Veierød MB. Pregnancy complications and birth outcomes among women experiencing nausea only or nausea and vomiting during pregnancy in the Norwegian Mother and Child Cohort Study. BMC Pregnancy Childbirth. 2015;15(1):138.

5. Mitsuda N, Eitoku M, Yamasaki K, Sakaguchi M, Yasumitsu-Lovell K, Maeda N, Fujieda M, Suganuma N. Nausea and vomiting during pregnancy associated with lower incidence of preterm births: the Japan Environment and Children's Study (JECS). BMC Pregnancy Childbirth. 2018;18(1):268.

6. Lindsay $\mathrm{KL}$, Buss $\mathrm{C}$, Wadhwa PD, Entringer S. The interplay between nutrition and stress in pregnancy: implications for fetal programming of brain development. Biol Psychiatry. 2019;85(2):135-49.

7. Chan JC, Nugent BM, Bale TL. Parental advisory: maternal and paternal stress can impact offspring neurodevelopment. Biol Psychiatry. 2018;83(10):886-94

8. Mackay E, Dalman C, Karlsson H, Gardner RM. Association of gestational weight gain and maternal body mass index in early pregnancy with risk for nonaffective psychosis in offspring. JAMA Psychiatry. 2017;74(4):339-49.

9. Neugebauer R, Hoek HW, Susser E. Prenatal exposure to wartime famine and development of antisocial personality disorder in early adulthood. Jama. 1999;282(5):455-62.

10. Parker SE, Starr JR, Collett BR, Speltz ML, Werler MM. Nausea and vomiting during pregnancy and neurodevelopmental outcomes in offspring. Paediatr Perinat Epidemiol. 2014;28(6):527-35.

11. Whitehouse AJ, Alvares GA, Cleary D, Harun A, Stojanoska A, Taylor LJ, Varcin KJ, Maybery M. Symptom severity in autism spectrum disorder is related to the frequency and severity of nausea and vomiting during pregnancy: a retrospective case-control study. Mol Autism. 2018;9(1):37.

12. Fejzo M, Kam A, Laguna A, MacGibbon K, Mullin P. Analysis of neurodevelopmental delay in children exposed in utero to hyperemesis gravidarum reveals increased reporting of autism spectrum disorder. Reprod Toxicol. 2019:84:59-64.

13. Getahun D, Fassett MJ, Jacobsen SJ, Xiang AH, Takhar HS, Wing DA, Peltier MR. Autism spectrum disorders in children exposed in utero to hyperemesis gravidarum. Am J Perinatol. 2019. https://doi.org/10.1055/s-0039-1696670. Epub ahead of print. PMID: 31581303

14. Rees S, Harding R. Brain development during fetal life: influences of the intra-uterine environment. Neurosci Lett. 2004;361(1-3):111-4.

15. Toro R, Leonard G, Lerner JV, Lerner RM, Perron M, Pike GB, Richer L, Veillette S, Pausova Z, Paus T. Prenatal exposure to maternal cigarette smoking and the adolescent cerebral cortex. Neuropsychopharmacology. 2008;33(5):1019.

16. Lebel C, Mattson SN, Riley EP, Jones KL, Adnams CM, May PA, Bookheimer SY, O'Connor MJ, Narr KL, Kan E. A longitudinal study of the long-term consequences of drinking during pregnancy: heavy in utero alcohol exposure disrupts the normal processes of brain development. J Neurosci. 2012;32(44):15243-51.

17. Buss C, Davis EP, Muftuler LT, Head K, Sandman CA. High pregnancy anxiety during mid-gestation is associated with decreased gray matter density in 69-year-old children. Psychoneuroendocrinology. 2010;35(1):141-53.

18. Arnsten AF, Rubia K. Neurobiological circuits regulating attention, cognitive control, motivation, and emotion: disruptions in neurodevelopmental psychiatric disorders. J Am Acad Child Adolesc Psychiatry. 2012;51(4):356-67.

19. Norman LJ, Carlisi C, Lukito S, Hart H, Mataix-Cols D, Radua J, Rubia K. Structural and functional brain abnormalities in attention-deficit/ hyperactivity disorder and obsessive-compulsive disorder: a comparative meta-analysis. JAMA Psychiatry. 2016;73(8):815-25.
20. Casey B, Cannonier T, Conley MI, Cohen AO, Barch DM, Heitzeg MM, Soules ME, Teslovich T, Dellarco DV, Garavan H. The adolescent brain cognitive development (ABCD) study: imaging acquisition across 21 sites. Dev Cogn Neurosci. 2018;32:43-54.

21. Bliddal M, Broe A, Pottegård A, Olsen J, Langhoff-Roos J. The Danish medical birth register. Eur J Epidemiol. 2018:33(1):27-36.

22. Lynge E, Sandegaard JL, Rebolj M. The Danish national patient register. Scand J Public Health. 2011;39(7_suppl):30-3.

23. Mors O, Perto GP, Mortensen PB. The Danish psychiatric central research register. Scand J Public Health. 2011;39(7_suppl):54-7.

24. Petersson F, Baadsgaard M, Thygesen LC. Danish registers on personal labour market affiliation. Scand J Public Health. 2011;39(7 suppl):95-8.

25. Schmidt M, Pedersen L, Sørensen HT. The Danish Civil Registration System as a tool in epidemiology. Eur J Epidemiol. 2014;29(8):541-9.

26. Schmidt M, Schmidt SAJ, Sandegaard JL, Ehrenstein V, Pedersen L, Sørensen HT. The Danish National Patient Registry: a review of content, data quality, and research potential. Clin Epidemiol. 2015;7:449.

27. Wallach Kildemoes H, Toft Sørensen H, Hallas J. The Danish national prescription registry. Scand J Public Health. 2011:39(7 suppl):38-41.

28. Auchter AM, Mejia MH, Heyser CJ, Shilling PD, Jernigan TL, Brown SA, Tapert SF, Dowling GJ. A description of the ABCD organizational structure and communication framework. Dev Cogn Neurosci. 2018;32:8-15.

29. Hagler DJ Jr, Hatton S, Cornejo MD, Makowski C, Fair DA, Dick AS, Sutherland MT, Casey BJ, Barch DM, Harms MP, et al. Image processing and analysis methods for the Adolescent Brain Cognitive Development Study. Neuroimage. 2019;202:116091.

30. Destrieux C, Fischl B, Dale A, Halgren E. Automatic parcellation of human cortical gyri and sulci using standard anatomical nomenclature. Neuroimage. 2010;53(1):1-15.

31. Gershon RC, Wagster MV, Hendrie HC, Fox NA, Cook KF, Nowinski CJ. NIH toolbox for assessment of neurological and behavioral function. Neurology. 2013;80(11 Supplement 3):S2-6.

32. Gershon RC, Slotkin J, Manly JJ, Blitz DL, Beaumont JL, Schnipke D, WallnerAllen K, Golinkoff RM, Gleason JB, Hirsh-Pasek K. IV. NIH toolbox cognition battery (CB): measuring language (vocabulary comprehension and reading decoding). Monogr Soc Res Child Dev. 2013:78(4):49-69.

33. Achenbach TM, Rescorla LA. Achenbach system of empirically based assessment. In: Volkmar FR, editor. Encyclopedia of autism spectrum disorders. New York: Springer New York; 2013. p. 31-9.

34. Gorham LS, Jernigan T, Hudziak J, Barch DM. Involvement in sports, hippocampal volume, and depressive symptoms in children. Biol Psychiatry. 2019:4(5):484-92.

35. Barch DM, Albaugh MD, Avenevoli S, Chang L, Clark DB, Glantz MD, Hudziak JJ, Jernigan TL, Tapert SF, Yurgelun-Todd D. Demographic, physical and mental health assessments in the adolescent brain and cognitive development study: rationale and description. Dev Cogn Neurosci. 2018;32: $55-66$.

36. Achenbach TM. International findings with the Achenbach System of Empirically Based Assessment (ASEBA): applications to clinical services, research, and training. Child Adolesc Psychiatry Ment Health. 2019;13(1):30

37. Achenbach TM, Rescorla LA. The Achenbach System of Empirically Based Assessment (ASEBA) for Ages 1.5 to 18 Years. Vol 2. 3rd ed. Mahwah: Erlbaum. 2004:179-213.

38. Garavan H, Bartsch H, Conway K, Decastro A, Goldstein R, Heeringa S, Jernigan T, Potter A, Thompson W, Zahs D. Recruiting the ABCD sample: design considerations and procedures. Deve Cogn Neurosci. 2018;32:16-22.

39. Jernigan $\mathrm{TL}$, Brown SA, Dowling GJ. The adolescent brain cognitive development study. J Res Adolesc. 2018;28(1):154-6.

40. Paulus MP, Squeglia LM, Bagot K, Jacobus J, Kuplicki R, Breslin FJ, Bodurka J, Morris AS, Thompson WK, Bartsch H, et al. Screen media activity and brain structure in youth: evidence for diverse structural correlation networks from the ABCD study. Neuroimage. 2019;185: 140-53.

41. Dick AS, Garcia NL, Pruden SM, Thompson WK, Hawes SW, Sutherland MT, Riedel MC, Laird AR, Gonzalez R. No evidence for a bilingual executive function advantage in the nationally representative ABCD study. Nat Hum Behav. 2019;3(7):692-701.

42. Cheng W, Rolls E, Gong W, Du J, Zhang J, Zhang X-Y, Li F, Feng J. Sleep duration, brain structure, and psychiatric and cognitive problems in children Mol Psychiatry. 2020. https://doi.org/10.1038/s41380-020-0663-2. Epub ahead of print. PMID: 32015467. 
43. Dick AS, Garcia NL, Pruden SM, Thompson WK, Hawes SW, Sutherland MT, Riedel MC, Laird AR, Gonzalez R. No evidence for a bilingual executive function advantage in the ABCD study. Nat Hum Behav. 2019;3(7):692-701.

44. Wager TD, Davidson ML, Hughes BL, Lindquist MA, Ochsner KN. Prefrontalsubcortical pathways mediating successful emotion regulation. Neuron. 2008;59(6):1037-50.

45. Lim SL, Padmala S, Pessoa L. Segregating the significant from the mundane on a moment-to-moment basis via direct and indirect amygdala contributions. Proc Natl Acad Sci U S A. 2009;106(39):16841-6.

46. Baron RM, Kenny DA. The moderator-mediator variable distinction in social psychological research: conceptual, strategic, and statistical considerations. J Pers Soc Psychol. 1986;51(6):1173-82.

47. Verberg M, Gillott D, Al-Fardan N, Grudzinskas J. Hyperemesis gravidarum, a literature review. Hum Reprod Update. 2005;11(5):527-39.

48. Dalsgaard S, McGrath J, Østergaard SD, Wray NR, Pedersen CB, Mortensen $\mathrm{PB}$, Petersen L. Association of mental disorder in childhood and adolescence with subsequent educational achievement. JAMA Psychiatry. 2020:e200217. https://doi.org/10.1001/jamapsychiatry.2020.0217. Epub ahead of print. PMID: 32211833.

49. Pedersen CB, Mors O, Bertelsen A, Waltoft BL, Agerbo E, McGrath JJ, Mortensen $\mathrm{PB}$, Eaton WW. A comprehensive nationwide study of the incidence rate and lifetime risk for treated mental disorders. JAMA Psychiatry. 2014;71(5):573-81.

50. Wang H, Li F, Miao M, Yu Y, Ji H, Liu H, Huang R, Obel C, Zhang J, Li J. Maternal spontaneous abortion and the risk of attention-deficit/ hyperactivity disorder in offspring: a population-based cohort study. Hum Reprod. 2020;35(5):1211-1221.

51. Remer J, Croteau-Chonka E, Dean DC III, D’Arpino S, Dirks H, Whiley D, Deoni SC. Quantifying cortical development in typically developing toddlers and young children, 1-6 years of age. Neuroimage. 2017;153:246-61.

52. Lyall AE, Shi F, Geng X, Woolson S, Li G, Wang L, Hamer RM, Shen D, Gilmore $\mathrm{JH}$. Dynamic development of regional cortical thickness and surface area in early childhood. Cereb Cortex. 2014;25(8):2204-12

53. Chaplin TA, Yu H-H, Soares JG, Gattass R, Rosa MG. A conserved pattern of differential expansion of cortical areas in simian primates. J Neurosci. 2013 33(38):15120-5.

54. Baroncelli L, Braschi C, Spolidoro M, Begenisic T, Sale A, Maffei L. Nurturing brain plasticity: impact of environmental enrichment. Cell Death Differ. 2010; 17(7):1092.

55. Fine JD, Moreau AL, Karcher NR, Agrawal A, Rogers CE, Barch DM, Bogdan R. Association of prenatal cannabis exposure with psychosis proneness among children in the adolescent Brain Cognitive Development (ABCD) Study. JAMA Psychiatry. 2019;76(7):762-764.

56. Peterson BS, Rauh VA, Bansal R, Hao X, Toth Z, Nati G, Walsh K, Miller RL, Arias $F$, Semanek D, et al. Effects of prenatal exposure to air pollutants (polycyclic aromatic hydrocarbons) on the development of brain white matter, cognition, and behavior in later childhood. JAMA Psychiatry. 2015;72(6):531-40.

57. Gautam P, Lebel C, Narr KL, Mattson SN, May PA, Adnams CM, Riley EP, Jones KL, Kan EC, Sowell ER. Volume changes and brain-behavior relationships in white matter and subcortical gray matter in children with prenatal alcohol exposure. Hum Brain Mapp. 2015;36(6):2318-29.

58. Rivkin MJ, Davis PE, Lemaster JL, Cabral HJ, Warfield SK, Mulkern RV, Robson CD, Rose-Jacobs R, Frank DA. Volumetric MRI study of brain in children with intrauterine exposure to cocaine, alcohol, tobacco, and marijuana. Pediatrics. 2008;121(4):741-50.

59. Woodward NC, Haghani A, Johnson RG, Hsu TM, Saffari A, Sioutas C, Kanoski SE, Finch CE, Morgan TE. Prenatal and early life exposure to air pollution induced hippocampal vascular leakage and impaired neurogenesis in association with behavioral deficits. Transl Psychiatry. 2018;8(1):261.

60. Sandman CA, Buss C, Head K, Davis EP. Fetal exposure to maternal depressive symptoms is associated with cortical thickness in late childhood. Biol Psychiatry. 2015;77(4):324-34.

61. Rolls ET. The cingulate cortex and limbic systems for emotion, action, and memory. Brain Struct Funct. 2019;224(9):3001-18.

62. Rolls ET, Cheng W, Gong W, Qiu J, Zhou C, Zhang J, Lv W, Ruan H, Wei D, Cheng K et al. Functional connectivity of the anterior cingulate cortex in depression and in health. Cereb Cortex. 2019;29:3617-30.

63. Rolls ET. The brain, emotion, and depression. Oxford: Oxford University Press; 2018.

64. Silk TJ, Beare R, Malpas C, Adamson C, Vilgis V, Vance A, Bellgrove MA. Cortical morphometry in attention deficit/hyperactivity disorder: contribution of thickness and surface area to volume. Cortex. 2016;82:1-10.
65. Cavanna AE, Trimble MR. The precuneus: a review of its functional anatomy and behavioural correlates. Brain. 2006;129(3):564-83.

66. Cheng W, Rolls ET, Gu H, Zhang J, Feng J. Autism: reduced functional connectivity between cortical areas involved in face expression, theory of mind, and the sense of self. Brain. 2015;138:1382-93.

67. Cheng W, Rolls ET, Qiu J, Yang D, Ruan H, Wei D, Zhao L, Meng J, Xie P, Feng J. Functional connectivity of the precuneus in unmedicated patients with depression. Biol Psychiatry Cogn Neurosci Neuroimaging. 2018;3(12): 1040-9.

68. Friedman LA, Rapoport JL. Brain development in ADHD. Curr Opin Neurobiol. 2015;30:106-11.

69. Apps MA, Rushworth MF, Chang SW. The anterior cingulate gyrus and social cognition: tracking the motivation of others. Neuron. 2016;90(4):692-707.

70. Gilbert SJ, Burgess PW. Executive function. Curr Biol. 2008;18(3):R110-4.

71. McLaughlin KA, Colich NL, Rodman AM, Weissman DG. Mechanisms linking childhood trauma exposure and psychopathology: a transdiagnostic model of risk and resilience. BMC Med. 2020;18:1-11.

72. Dennis EL, Thompson PM. Typical and atypical brain development: a review of neuroimaging studies. Dialogues Clin Neurosci. 2013;15(3):359.

73. Hoogman M, van Rooij D, Klein M, Boedhoe P, llioska I, Li T, Patel Y, Postema MC, Zhang-James Y, Anagnostou E. Consortium neuroscience of attention deficit/hyperactivity disorder and autism spectrum disorder: the ENIGMA adventure. Hum Brain Mapp. 2020. https://doi.org/10.1002/hbm. 25029. Epub ahead of print. PMID: 32420680.

74. Du JRE, Gong W, Cao M, Vatansever D, Cheng W, Feng J. Association between parental age, brain structure, and psychiatric and cognitive problems in children; 2020.

75. McCarthy FP, Khashan AS, North RA, Moss-Morris R, Baker PN, Dekker G, Poston L, Kenny LC, consortium S. A prospective cohort study investigating associations between hyperemesis gravidarum and cognitive, behavioural and emotional well-being in pregnancy. PLoS One. 2011;6(11):e27678.

76. Petraglia F, Sutton S, Vale W. Neurotransmitters and peptides modulate the release of immunoreactive corticotropin-releasing factor from cultured human placental cells. Am J Obstet Gynecol. 1989;160(1):247-51.

77. O'donnell K, O'connor T, Glover V. Prenatal stress and neurodevelopment of the child: focus on the HPA axis and role of the placenta. Dev Neurosci. 2009;31(4):285-92.

78. Howland MA, Sandman CA, Glynn LM, Crippen C, Davis EP. Fetal exposure to placental corticotropin-releasing hormone is associated with child selfreported internalizing symptoms. Psychoneuroendocrinology. 2016;67:10-7.

79. Sandman CA, Curran MM, Davis EP, Glynn LM, Head K, Baram TZ. Cortical thinning and neuropsychiatric outcomes in children exposed to prenatal adversity: a role for placental CRH? Am J Psychiatr. 2018;175(5):471-9.

80. Van den Bergh BR, van den Heuvel Ml, Lahti M, Braeken M, de Rooij SR, Entringer S, Hoyer D, Roseboom T, Räikkönen K, King S. Prenatal developmental origins of behavior and mental health: the influence of maternal stress in pregnancy. Neurosci Biobehav Rev. 2017:S01497634(16)30734-5. https://doi.org/10.1016/j.neubiorev.2017.07.003. Epub ahead of print. PMID: 28757456.

81. Van den Bergh BR, Van Calster B, Smits T, Van Huffel S, Lagae L. Antenatal maternal anxiety is related to HPA-axis dysregulation and self-reported depressive symptoms in adolescence: a prospective study on the fetal origins of depressed mood. Neuropsychopharmacology. 2008;33(3):536-45.

82. Tamura M, Sajo M, Kakita A, Matsuki N, Koyama R. Prenatal stress inhibits neuronal maturation through downregulation of mineralocorticoid receptors. J Neurosci. 2011;31(32):11505-14.

83. Attard CL, Kohli MA, Coleman S, Bradley C, Hux M, Atanackovic G, Torrance GW. The burden of illness of severe nausea and vomiting of pregnancy in the United States. Am J Obstet Gynecol. 2002;186(5):S220-7.

84. Plana-Ripoll O, Pedersen CB, Agerbo E, Holtz Y, Erlangsen A, Canudas-Romo V, Andersen PK, Charlson FJ, Christensen MK, Erskine HE. A comprehensive analysis of mortality-related health metrics associated with mental disorders: a nationwide, register-based cohort study. Lancet. 2019;394(10211):1827-35.

85. Lawlor DA, Tilling K, Davey Smith G. Triangulation in aetiological epidemiology. Int J Epidemiol. 2016;45(6):1866-86.

\section{Publisher's Note}

Springer Nature remains neutral with regard to jurisdictional claims in published maps and institutional affiliations. 